\title{
NUMERICAL SIMULATION OF ELECTRIC CURRENTS THROUGH INSULATING MATERIALS
}

\author{
F. Frutos, A. Jiménez and J.A. Pérez \\ Dpto. Física Aplicada. Facultad de Informática. Universidad de Sevilla, Spain
}

\begin{abstract}
A numerical simulation of electric currents is carried out through solid dielectrics from a model based on the local polarization. A degradation of material was considered to be produced from one of the electrodes and we simulated it by admitting modifications in the chemical structure of the material, which implies an alteration in the value of the corresponding dipole moments that characterize the material, as well as the concentration of the latest. Different simulations are achieved according to the ratio of the degraded thickness and the temperature.
\end{abstract}

\section{INTRODUCTION}

The main objective of this work has been to know qualitatively how the polarization current behaves, which can be obtained from a solid dielectric with features such as lineal dipolar phenomenons. To obtain this, such current must be calculated by means of a simple model in order to prove the basic dependence that this current presents due to temperature variations and the material degradation.

In future works this preliminary study will be completed with the introduction of more complex polarization processes.

\section{PHYSICAL MODEL}

As it is known, lots of researchers have suggested that the orientation of dipoles is responsible for the polarization currents process in some dielectric solids.

Nevertheless, for other dielectric materials where there are not any prominent dipolar groups the polarization currents could be due to ions jumps between close equilibrium positions. Both phenomenons can be described from a model based on two possible hypotheses. The first suggest that the presence of an electric field causes a twisting of molecular chains. This last implies a redistribution of the dipole moments and accordingly, a change of the local polarization with time. The second hypothesis is based on the possibility of ions jumps between equilibrium positions separated by potential barriers. The presence of an electrical field causes a redistribution in the concentrations of such ions, appearing a dipole moment which results in another contribution to change the local polarisation with time.

In the simplified case in which only two dipoles orientations might be possible, which is equivalent to the ions movement between the equilibrium positions of a two potential well, it is easy to show $[1,2]$ that the variation of the local polarisation $P$ with the time in a sample of plane geometry where the surface $S$ is much bigger than thickness $L$, is,

$\frac{\partial P(x, t)}{\partial t}=-\alpha \cosh \left(\frac{p E(x, t)}{k T}\right) P(x, t)+\alpha n p s e n h\left(\frac{p E(x, t)}{k T}\right)$

where $\alpha$ is proportional to the probability per second that one ion changes its equilibrium position, with the absence of an electric field, due to the thermal energy and is expressed by,

$$
\alpha=2 v \exp \left(-\frac{W}{k T}\right)
$$

where $v$ is the vibration frequency of the ions within their wells, and $W$ is the potential barrier height which separates the two respective well sites, $E(x, t)$ is the electric field, $n$ is the concentration of ions, $p$ the dipolar moment associated to one ion.

In the case that $p E$ is far minor than $k T$, the equation (1) is reduced to,

$$
\frac{\partial P(x, t)}{\partial t}=-\alpha P(x, t)+\frac{\alpha b(x)}{k T} E(x, t)
$$

where,

$b(x)=p^{2} n(x)$

From (3) on the current density $J(t)$ is calculated by,

$J(t)=\varepsilon_{0} \frac{\partial E(x, t)}{\partial t}+\frac{\partial P(x, t)}{\partial t}$

where the ohmic conduction term is rejected.

If we consider a $V_{a} \cdot u\left(t-t_{a}\right)$ step voltage applied to the sample, the equation (3) can be solved analytically for an homogeneous sample, and the result is given by, 


$$
P(t)=\frac{V_{a}}{L} \frac{b}{k T}\left[1-\exp \left(-\alpha\left(t-t_{a}\right)\right)\right]
$$

In this case, the current density obtained from (5) and (6) is,

$$
J(t)=\frac{V_{a}}{L}\left[\varepsilon_{o} \delta\left(t-t_{a}\right)+\frac{b}{k T} \alpha \exp \left(-\alpha\left(t-t_{a}\right)\right)\right]
$$

In the case that the material is heterogeneous and consequently degraded, it is not possible, in general, to obtain an analytical solution from (3). For that we used numerical methods to calculate the polarization $P(x, t)$ and the polarization current.

\section{CALCULATIONS}

The following hypotheses has been admitted in the numerical calculus: 1) The parameter $\alpha$ is independent from the position. 2) The parameter $b$, which is related with the permittivity of the medium, depends on position according to this model,

$b(x)= \begin{cases}a-m x, & x \in[0, g L] \\ b_{h}, & x \in[g L, L]\end{cases}$

where $g$ represents the degraded material ratio, being $a$ the $b$ value corresponding to a maximum permittivity of 3.7 in the degradation origin and $b_{h}$ the $b$ value in the undegraded area, where the permittivity is 2.3 . This model has been chosen (8) based on experimental measurements of the permittivity in the polyethylene with water trees [2].

\section{RESULTS}

The polarization current has been determined in relation to time, temperature and the ratio, $g$, of the degraded material. The Fig.1 shows the results for an homogeneous sample, undegraded, for several temperatures. We observed how the initial current values increase when the temperature is also increased at the beginning, but the currents decrease faster with time.

In Fig. 2 the polarization currents are represented in relation to time taking as parameter the ratio of degraded material $g$ for $300 \mathrm{~K}$ constant temperature. A decrease of the current can be observed when the degradation is increased. In all cases a cuasi-exponential diminution is shown. In fact, if the material is homogeneous, $g=0$, the diminution will be exponential such as figures 2 and 1 . This behaviour was expected, because of the chosen physical model.

In Fig. 3 are represented the polarisation currents being the exact instant $t=100$ seconds in relation to temperature for an homogeneous sample and three ratios of degradation. We observe that the maximum seems independent from the degradation ratio.

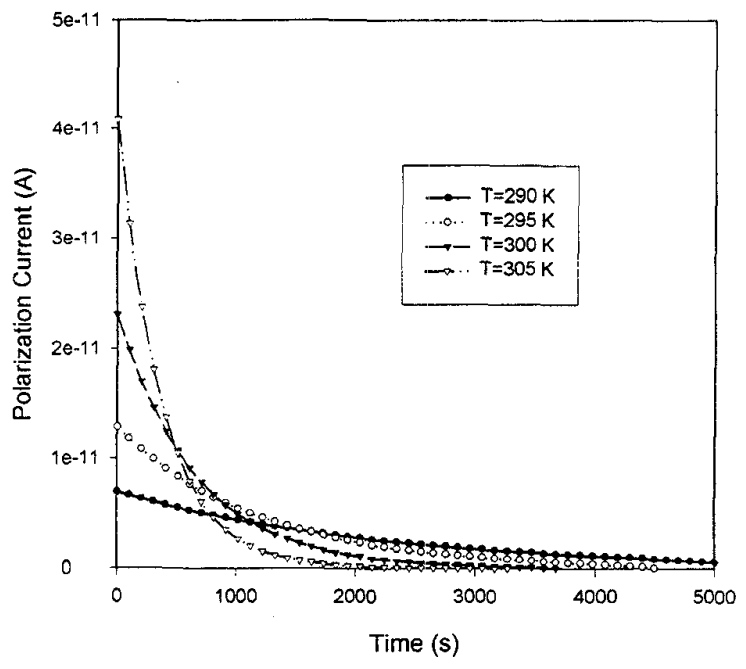

Fig. 1. Polarisation current versus time for an homogeneous material for several values of $T$.

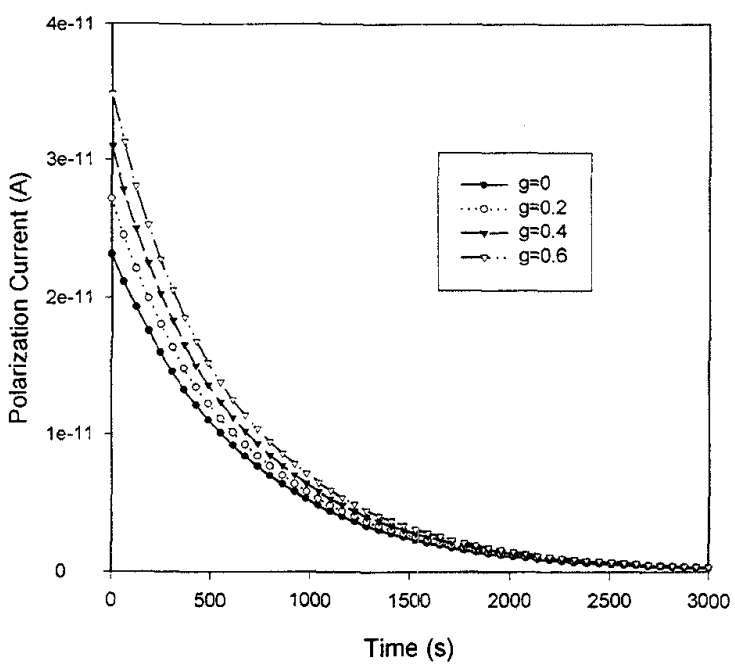

Fig. 2 Polarisation current versus time for several values of $g$ for $T=300 \mathrm{~K}$.

In Fig. 4 we show the movement derived from the maximum (mentioned above in Fig.3) with the temperature depending on the moment the measurement is taken.

The current increases in the initial moment with the temperature for different ratios of degradation is observed in Fig. 7.

The time taken for the current to be reduced to its half in relation to the temperature for different ratios of degradation is shown in Fig. 5 


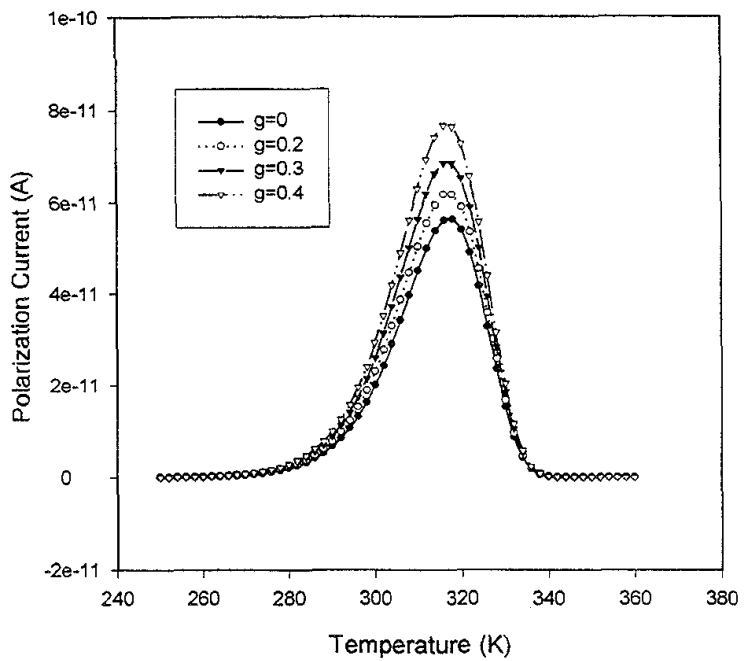

Fig. 3. Polarization current value in the exact instant $t=100 \mathrm{~s}$. versus the temperature for different $\mathrm{g}$ degradation ratios.

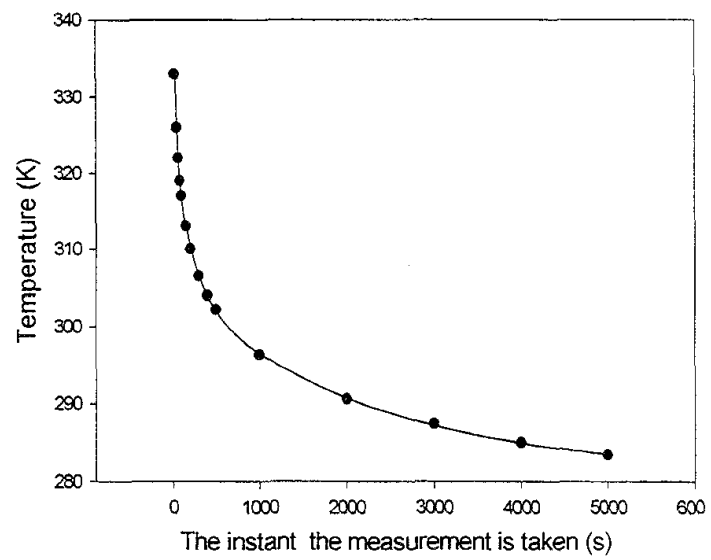

Fig. 4 Evolution of the position for the highest polarisation current with the instant the measurement is taken.

The value of the electric field in the interior of a sample with $800 \mu \mathrm{m}$ thickness for three degradation values and an applied voltage of $2.5 \mathrm{kV}$ is shown in Fig. 6 . We can observe how the field is minimum close to the degraded area and it grows to the utmost value in the undegraded area. At the same time that the degradation ratio increases, the value of the field is more considerable. For the case of $g=0.6$ the field amplification is between $80-85 \%$.

\section{DISCUSSION}

Currents increase with the material degradation is observed in Fig.2. This is due to the fact that the higher the degradation is, the higher the average of the $b(x)$ function is as a result of the dipoles concentration by the square of the dipolar moment of the molecules. On the

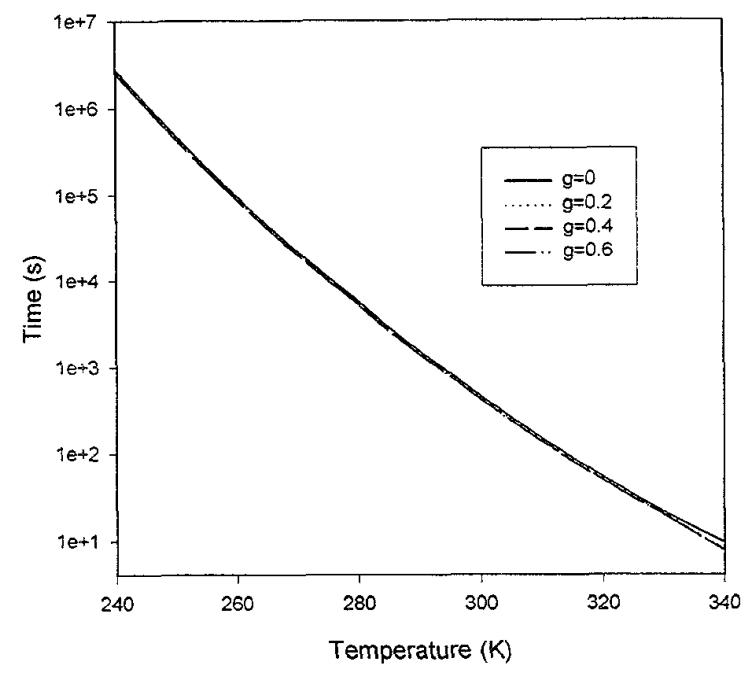

Fig. 5. Fall time evolution to half of the polarisation current versus the temperature for different $g$ degradation ratios.

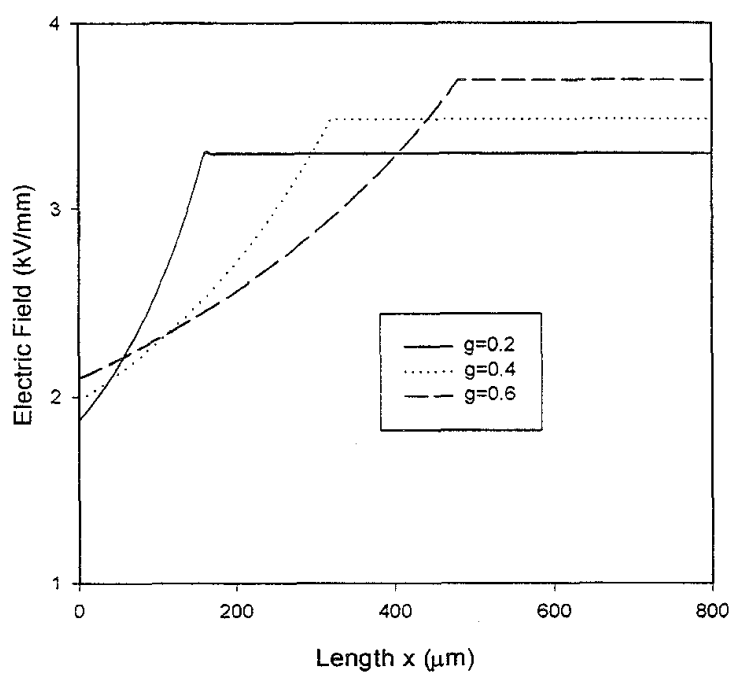

Fig.6 Electric field mapping in an $800 \mu \mathrm{m}$ thick sample subjected to a voltage of $2.5 \mathrm{kV}$ for different $\mathrm{g}$ degradation ratios

other hand, the polarisation current in the initial instant increases with the temperature as it is seen in Fig.1. Nevertheless, the decay time of such a current decreases considerably with the temperature and some time later the currents derived from a given temperature surpass those ones with the highest temperatures. The explanation could be that the higher temperature is the faster ions transitions are produced. This behaviour could also explain the plot that is obtained when showing the temperature for a fixed instant $(t=100 \mathrm{~s}$. in Fig. 3). The maximum observed corresponds to the optimal conditions in which the effect of increase of the initial current is combined with the decrease of the 
decay time with the temperature increase. In fact, the positions of the maximum depend on the chosen instant to carry out the measurements, as it is seen on Fig. 4.

The current increases with the degradation as we observe in Fig.3, which is also shown in Fig.2. The dependence in relation to the ratio degradation is not lineal as it is also shown in Fig. 7.

The time in which the current decays to half of its initial value decreases with the temperature, as it is shown in Fig. 5 and the degradation does not influence greatly this decay time.

A study of the electric field has been carried out within the material for different degradations. As a result we observed an amplification effect. The more degraded the material was, the higher the amplification was, as is shown in Fig.6.

This effect could be explained because in the most degraded area (more polarizable) a distribution of polarisation charge higher than in the undegraded area is generated. Such a charge neutralizes more effectively the external field; therefore the total field in that area is lower. This causes the field in the undegraded area to be amplified in order to keep the field integral equal to the applied tension.

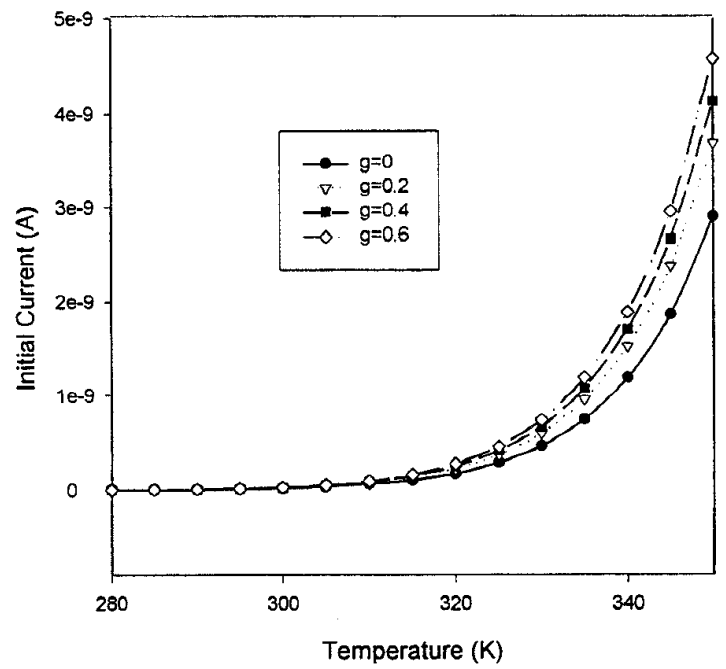

Fig.7 Initial current variation versus the temperature for different degradation values.

\section{CONCLUSIONS}

The above studied model tries to make a qualitative and very simplified description of the real systems.

In the future other mechanisms must be considered together with the presented model.

On the other hand, we have avoided to make an hypothesis about the exact meaning of the function $b(x)$, where two microscopic parameters are involved: The individual dipolar moment of every dipole and the concentration of them. If we explicitly take them into consideration, we will be able to integrate the equation (1) with no need of approximation a priori.

Lastly, the results of the simulation should be contrasted with experience to register all the similarities and divergences that will give us information to modify the model properly.

\section{REFERENCES}

[1] R. Bartnikas, "Engineering Dielectrics", vol II, eds Bartnikas/Eichhorn, (ATSM Press, Philadelphia, 1979.

[2] A.K. Jonscher, "Dielectric Relaxation in Solids". Chelsea Dielectric Press Ld. London, 1983.

[3] I. Radu, et al. "A study on the dependence of water tree permittivity with time", Annual Report CEIDP, pp $762-765,1996$. 